The Chittagong Univ. J. Sci. 42(1): 39-57, 2020

\title{
Anisotropic Universe with Minimally Interacting Matter and Holographic Dark Energy
}

\author{
Mohammad Moksud Alam \\ Department of Mathematics, University of Chittagong, Chittagong-4331, Bangladesh \\ E-mail: moksud.math@cu.ac.bd
}

Manuscript received on 29 December, 2019, Revised manuscript received on 16 August, 2020 and accepted on 01 December, 2020.

\begin{abstract}
The holographic dark energy (HDE), a form of dark energy, has been a useful tool in explaining the recent phase transition of the universe. In this paper, we study the anisotropic and homogeneous Bianchi type-III model of the universe filled with minimally interacting matter and holographic dark energy under the framework of the Brans-Dicke (BD) scalar tensor theory of gravitation. Considering two physically plausible conditions such as, (i) the special law of variation for Hubble parameter and (ii) the scalar expansion proportional to the shear scalar, we propose two new models namely, exponential expansion model and power law expansion model. We also show the dynamics of these models fit with existing observational data and literature thereof. The transit behavior of the equation of state parameter for dark energy has been analyzed graphically. The jerk parameter is also studied for both of the models describing cosmological evolution.
\end{abstract}

Keywords: Holographic dark energy (HDE), Bianchi type-III model, Brans-Dicke theory, Jerk parameter.

DOI: https://doi.org/10.3329/cujs.v42i1.54237

হনোগ্রাফিক গুপ্ত শক্তি, এক ধরণের গুপ্ত শক্তি, যা মহাবিশ্বের সাম্প্রতিক দশা রূপান্তর ব্যাখা করতে একটি কার্যকর পদ্ধতি। এ গবেষণাপত্রে, মহাকর্ষের উপর ব্রান্স-ডিঁকি স্কেলার টেনসর তত্ত্বের কাঠামোতে ন্যূনতমরূপে ক্রিয়াশীল পদার্থ এবং হলোগ্রাফিক গুপ্ত শক্তিতে পূর্ণ মহাবিশ্বের অ্যানাইলেট্রপিক ও সমশ্রেণীভুক্ত বিয়াঙ্কি টাইপ-III মডেল অধ্যয়ন করি। বাস্তব যুক্তিসংগত দ্রুটি শর্ত যথা (১) হাবল প্যারামিটার পরিবর্তনের বিশেষ বিধি এবং (২) স্কেলার সম্প্রসারণ শিয়ার স্কেলারের সমানুপাতিক, বিবেচনা করে আমরা দ্রুটি নতুন মডেল প্রস্তাব করি যথা- সূচকীয় সম্প্রসারণ মডেল এবং ঘাত বিধি সম্প্রসারণ মডেল। আমরা আরও দেখাই যে মডেলগুলোর গতিশীলতা বিদ্যমান পর্যবেক্ষণমূলক তথ্য এবং বিদ্যার সাথে সঙ্গতিপূর্ণ। গুপ্ত শক্তির জন্য অবস্থার সমীকরণের প্যারামিটার রুপান্তরের ধরণ চিত্রসহকারে ব্যাখা করা হয়। মহাজাগতিক বিবর্তন বর্ণনা করতে উভয় মডেলের জার্ক প্যারামিটারও অধ্যয়ন করা হয়। 
Mohammad Moksud Alam

\section{Introduction}

Recent observational studies very rationally explain the accelerated expansion of the current universe [1-5]. It seems to have some mysterious energy behind this expansion which is pulling apart all the cosmological objects. The nature of such entity brings it a name "dark energy". Observational data of standard cosmology shows that dark energy occupies about $73 \%$ of the energy of our universe that exerts a large negative pressure and dark matter occupies about $23 \%$ whereas the ordinary matter occupies only about $4 \%$ of the total energy of the universe. Thus our present conviction of the universe is that it contains cosmic fluid consisting of dark matter and dark energy with independent evolution.

In recent years, several models have been proposed satisfying the present ratio of the dark energy. Despite having a large discrepancy with different theories and observations, the cosmological term is assumed to be the most simple and natural candidate for explaining dark energy and cosmic acceleration since it fits into the observational data [6, 7]. In recent time, modified theories of gravitation are attracting more and more attention to explain early inflation and late time acceleration and dark energy. Some significant modified theories of gravity includes scalar-tensor theories of gravity proposed in $[8,9], f(R)$ theory of gravity [10] and $f(R, T)$ gravity [11].

In 1961, Brans and Dicke [8] brought an interesting alternative to general relativity based on Mach's principle. Brans-Dicke (BD) theory is explained by a scalar function $\varphi$ which has the dimensions of the inverse of gravitational constant and interacts equally with all forms of matter. Due to its vast cosmological implications, the BD theory is considered as a significant scalar-tensor theory of gravitation [12,13], based on which the latest inflationary models [14], possible 'graceful exit problem' [15] and extended chaotic inflation [16] have been investigated. 
Attention paid to the holographic dark energy models is the best choice among the many different approaches explaining dark energy cosmological models. The holographic dark energy model, constructed by the holographic principle [17], is an emerging model as a candidate for dark energy. It is considered that the cosmological constant problem and some other issues may be interpreted by this model. Cohen et al. [18], Horova and Minic [19], Thomas [20], Hsu [21] and Li [22] have investigated several aspects of holographic dark energy. In a study [23], Setare and Vagenas have discussed the cosmological dynamics of the interacting holographic dark energy model. Das and Mammon [24] discussed interacting model of dark energy in the BD theory. In current time, Sarkar and Mahanta [25] and Sarkar [26, 27] have investigated minimally interacting holographic dark energy models in the Bianchi type-I universe, while Adhav et al. [28] have studied the interacting dark matter and the holographic dark energy in an anisotropic universe. Very recently, Kiran et al. [29] obtained Bianchi type-V minimally interacting holographic dark energy model for the scalar-tensor theory of gravitation given by Saez and Ballester [9].

This paper is devoted to investigate the exact solutions of Bianchi type III spacetime in the context of Brans-Dicke scalar tensor theory of gravitation. For this, we assume two physically plausible conditions such as, (i) a law of variation of Hubble's parameter and (ii) the scalar expansion is proportional to the shear scalar, in Bianchi type III space-time model of the universe filled with minimally interacting dark matter and holographic dark energy. These conditions together with field equations lead to two different solutions of the models representing exponential expansion model and power law expansion model.

\section{Basic Equations}

In this section, we study the BD field equations with the help of Bianchi type-III metric in the presence of matter and holographic dark energy [30-32]. We take the Bianchi type-III spatially homogeneous and anisotropic space-time metric defined by 
$d s^{2}=d t^{2}-a_{1}^{2}(t) d x^{2}-a_{2}^{2}(t) e^{-2 \alpha x} d y^{2}-a_{3}^{2}(t) d z^{2}$

where $\alpha$ is a positive constant which can be taken as unity.

And the Brans-Dicke (BD) field equations [8] for combined scalar and tensor fields in the presence of dark matter and holographic dark energy as follows

$$
R_{i j}-\frac{1}{2} g_{i j} R+\frac{\omega}{\varphi^{2}}\left(\varphi_{, i} \varphi_{, j}-\frac{1}{2} g_{i j} \varphi_{, k} \varphi^{, k}\right)+\frac{1}{\varphi}\left(\varphi_{; i j}-g_{i j} \varphi_{; k}^{, k}\right)=-8 \pi \varphi^{-1}\left(T_{i j}+\bar{T}_{i j}\right)
$$

where $\varphi$ is the scalar field satisfying

$$
\varphi_{; k}^{, k}=8 \pi(3+2 \omega)^{-1}(T+\bar{T})
$$

Moreover, the energy conservation for dark matter and holographic dark energy is confined by

$T_{; j}^{i j}+\bar{T}_{; j}^{i j}=0$

where $R_{i j}$ is the Ricci tensor, $R$ is the Ricci scalar, $\omega$ is the dimensionless coupling constant (constrained as $\omega \geq 40000$ for its consistency with solar system bounds $[33,34]), \varphi$ is the BD scalar field, and $T_{i j}$ and $\bar{T}_{i j}$ are energy momentum tensors for dark matter and holographic dark energy, respectively, defined as

$$
\begin{aligned}
& T_{i j}=\rho_{m} u_{i} u_{j} \\
& \bar{T}_{i j}=\left(\rho_{\lambda}+p_{\lambda}\right) u_{i} u_{j}-g_{i j} p_{\lambda}
\end{aligned}
$$

Thus in a case of co-moving coordinate system with the metric defined by (1), the BD field equations (2) and (3) are transformed into the followings

$$
\begin{aligned}
& \frac{\ddot{a}_{2}}{a_{2}}+\frac{\ddot{a}_{3}}{a_{3}}+\frac{\dot{a}_{2} \dot{a}_{3}}{a_{2} a_{3}}+\frac{\omega}{2} \frac{\dot{\varphi}^{2}}{\varphi^{2}}+\frac{\ddot{\varphi}}{\varphi}+\frac{\dot{\varphi}}{\varphi}\left(\frac{\dot{a}_{2}}{a_{2}}+\frac{\dot{a}_{3}}{a_{3}}\right)=-8 \pi \varphi^{-1} p_{\lambda} \\
& \frac{\ddot{a}_{1}}{a_{1}}+\frac{\ddot{a}_{3}}{a_{3}}+\frac{\dot{a}_{1} \dot{a}_{3}}{a_{1} a_{3}}+\frac{\omega}{2} \frac{\dot{\varphi}^{2}}{\varphi^{2}}+\frac{\ddot{\varphi}}{\varphi}+\frac{\dot{\varphi}}{\varphi}\left(\frac{\dot{a}_{1}}{a_{1}}+\frac{\dot{a}_{3}}{a_{3}}\right)=-8 \pi \varphi^{-1} p_{\lambda} \\
& \frac{\ddot{a}_{1}}{a_{1}}+\frac{\ddot{a}_{2}}{a_{2}}+\frac{\dot{a}_{1} \dot{a}_{2}}{a_{1} a_{2}}-\frac{1}{a_{1}^{2}}+\frac{\omega}{2} \frac{\dot{\varphi}^{2}}{\varphi^{2}}+\frac{\ddot{\varphi}}{\varphi}+\frac{\dot{\varphi}}{\varphi}\left(\frac{\dot{a}_{1}}{a_{1}}+\frac{\dot{a}_{2}}{a_{2}}\right)=-8 \pi \varphi^{-1} p_{\lambda}
\end{aligned}
$$




$$
\begin{aligned}
& \frac{\dot{a}_{1} \dot{a}_{2}}{a_{1} a_{2}}+\frac{\dot{a}_{1} \dot{a}_{3}}{a_{1} a_{3}}+\frac{\dot{a}_{2} \dot{a}_{3}}{a_{2} a_{3}}-\frac{1}{a_{1}^{2}}-\frac{\omega}{2} \frac{\dot{\varphi}^{2}}{\varphi^{2}}+\frac{\dot{\varphi}}{\varphi}\left(\frac{\dot{a}_{1}}{a_{1}}+\frac{\dot{a}_{2}}{a_{2}}+\frac{\dot{a}_{3}}{a_{3}}\right)=8 \pi \varphi^{-1}\left(\rho_{m}+\rho_{\lambda}\right) \\
& \frac{\dot{a}_{1}}{a_{1}}-\frac{\dot{a}_{2}}{a_{2}}=0
\end{aligned}
$$

And also, we derive the following expression for the BD scalar field using the Bianchi type-III metric

$$
\varphi_{; k}^{, k}=\ddot{\varphi}+\dot{\varphi}\left(\frac{\dot{a}_{1}}{a_{1}}+\frac{\dot{a}_{2}}{a_{2}}+\frac{\dot{a}_{3}}{a_{3}}\right)
$$

Plugging this value in equation (3), we obtain

$$
\ddot{\varphi}+\dot{\varphi}\left(\frac{\dot{a}_{1}}{a_{1}}+\frac{\dot{a}_{2}}{a_{2}}+\frac{\dot{a}_{3}}{a_{3}}\right)=8 \pi(3+2 \omega)^{-1}\left(\rho_{m}+\rho_{\lambda}-\rho_{\lambda}\right)
$$

where an overhead dot (.) denotes the differentiation with respect to cosmic time $t$. In the study of holographic dark energy, Barotropic equation of state is given by

$$
p_{\lambda}=w \rho_{\lambda}
$$

where $w$ is the equation of state (EoS) parameter for the dark energy. The vacuum energy $(w=-1)$ is the simplest dark energy (DE) candidate, which is assumed to be equivalent to the cosmological term $(\Lambda)$ [35]. Based on the evolution, quintessence $(w>-1)$, phantom $(w<-1)$ and quintom (transition from $w<-1$ to $w>-1)$ are some of the usual alternatives, explaining minimally coupled scalar fields.

Taking the covariant differentiation of the energy momentum tensor for dark matter and holographic dark energy (HDE), we obtain, respectively

$$
\begin{aligned}
& T_{; j}^{i j}=\dot{\rho}_{m}+\left(\frac{\dot{a}_{1}}{a_{1}}+\frac{\dot{a}_{2}}{a_{2}}+\frac{\dot{a}_{3}}{a_{3}}\right) \rho_{m} \text { and } \\
& \bar{T}_{; j}^{i j}=\dot{\rho}_{\lambda}+\left(\frac{\dot{a}_{1}}{a_{1}}+\frac{\dot{a}_{2}}{a_{2}}+\frac{\dot{a}_{3}}{a_{3}}\right)(1+w) \rho_{\lambda}
\end{aligned}
$$


44 Mohammad Moksud Alam

Using these results, we write the equation of conservation (4) for matter and dark energy as follows

$\dot{\rho}_{m}+\left(\frac{\dot{a}_{1}}{a_{1}}+\frac{\dot{a}_{2}}{a_{2}}+\frac{\dot{a}_{3}}{a_{3}}\right) \rho_{m}+\dot{\rho}_{\lambda}+\left(\frac{\dot{a}_{1}}{a_{1}}+\frac{\dot{a}_{2}}{a_{2}}+\frac{\dot{a}_{3}}{a_{3}}\right)(1+w) \rho_{\lambda}=0$

In this study, we consider the minimally interacting matter and holographic dark energy. Both of the components are conserved separately so that we have $[26,27]$

$\dot{\rho}_{m}+\rho_{m}\left(\frac{\dot{a}_{1}}{a_{1}}+\frac{\dot{a}_{2}}{a_{2}}+\frac{\dot{a}_{3}}{a_{3}}\right)=0$ and
$\dot{\rho}_{\lambda}+\left(\frac{\dot{a}_{1}}{a_{1}}+\frac{\dot{a}_{2}}{a_{2}}+\frac{\dot{a}_{3}}{a_{3}}\right)(1+w) \rho_{\lambda}=0$

To study the field equations above, let us define the parameters for the metric (1) as given below.

The spatial volume is

$V=a_{1} a_{2} a_{3}=a^{3}(t)$

where $a(t)$ is the average scale factor of the universe.

and the directional Hubble parameters are

$H_{1}=\frac{\dot{a}_{1}}{a_{1}}, \quad H_{2}=\frac{\dot{a}_{2}}{a_{2}}, \quad H_{3}=\frac{\dot{a}_{3}}{a_{3}}$

with the average Hubble parameter given by

$H=\frac{1}{3}\left(H_{1}+H_{2}+H_{3}\right)$

Also, the expression for scalar expansion $\theta$ and shear scalar $\sigma^{2}$ are

$\theta=u_{; i}^{, i}=3 H=\frac{\dot{a}_{1}}{a_{1}}+\frac{\dot{a}_{2}}{a_{2}}+\frac{\dot{a}_{3}}{a_{3}}$

$\sigma^{2}=\frac{1}{2} \sigma^{i j} \sigma_{i j}=\frac{1}{2}\left[\left(\frac{\dot{a}_{1}}{a_{1}}\right)^{2}+\left(\frac{\dot{a}_{2}}{a_{2}}\right)^{2}+\left(\frac{\dot{a}_{3}}{a_{3}}\right)^{2}-\frac{\dot{a}_{1} \dot{a}_{2}}{a_{1} a_{2}}-\frac{\dot{a}_{2} \dot{a}_{3}}{a_{2} a_{3}}-\frac{\dot{a}_{1} \dot{a}_{3}}{a_{1} a_{3}}\right]$ 
The average anisotropy parameter is defined as

$$
\Delta=\frac{1}{3} \sum_{i=1}^{3}\left(\frac{H_{i}-H}{H}\right)^{2}
$$

where $H_{i}(i=1,2,3)$ represent the directional Hubble parameters.

\section{Bianchi type-III Universe in Brans-Dicke Theory}

In this section, we present the solutions of the set of equations (7) - (12) in the minimally interacting matter and holographic dark energy model in the Bianchi type III universe in Brans-Dicke theory. Then taking the special law of variation for Hubble parameter into account [36], we present two new models for zero and nonzero values of $n$.

A simple integration of the equation (11) leads to

$$
a_{1}=l a_{2}
$$

where $l$ is a constant of integration, which can be chosen as unity without loss of generality, so that we have

$$
a_{1}=a_{2}
$$

Now using equation (24), set of equations (7)-(10) and (12) reduce to the following independent equations

$$
\begin{aligned}
& \frac{\ddot{a}_{2}}{a_{2}}+\frac{\ddot{a}_{3}}{a_{3}}+\frac{\dot{a}_{2} \dot{a}_{3}}{a_{2} a_{3}}+\frac{\omega}{2} \frac{\dot{\varphi}^{2}}{\varphi^{2}}+\frac{\ddot{\varphi}}{\varphi}+\frac{\dot{\varphi}}{\varphi}\left(\frac{\dot{a}_{2}}{a_{2}}+\frac{\dot{a}_{3}}{a_{3}}\right)=-8 \pi \varphi^{-1} p_{\lambda} \\
& 2 \frac{\ddot{a}_{2}}{a_{2}}+\frac{\dot{a}_{2}^{2}}{a_{2}^{2}}-\frac{1}{a_{2}^{2}}+\frac{\omega}{2} \frac{\dot{\varphi}^{2}}{\varphi^{2}}+\frac{\dot{\varphi}}{\varphi}\left(2 \frac{\dot{a}_{2}}{a_{2}}\right)=-8 \pi \varphi^{-1} p_{\lambda} \\
& \frac{\dot{a}_{2}^{2}}{a_{2}^{2}}+2 \frac{\dot{a}_{2} \dot{a}_{3}}{a_{2} a_{3}}-\frac{1}{a_{2}^{2}}-\frac{\omega}{2} \frac{\dot{\varphi}^{2}}{\varphi^{2}}+\frac{\dot{\varphi}}{\varphi}\left(2 \frac{\dot{a}_{2}}{a_{2}}+\frac{\dot{a}_{3}}{a_{3}}\right)=8 \pi \varphi^{-1}\left(\rho_{m}+\rho_{\lambda}\right) \\
& \ddot{\varphi}+\dot{\varphi}\left(2 \frac{\dot{a}_{2}}{a_{2}}-\frac{\dot{a}_{3}}{a_{3}}\right)=8 \pi(3+2 \omega)^{-1}\left(\rho_{m}+\rho_{\lambda}-3 p_{\lambda}\right)
\end{aligned}
$$


These equations form a system of four independent equations with five unknowns $a_{2}, a_{3}, \varphi, \rho_{\lambda}, p_{\lambda}$ [since $\rho_{m}$ can be eliminated by equation (15)]. To reach a deterministic solution, we adopt the following three physically plausible conditions. (i) The shear scalar $\sigma^{2}$ is proportional to scalar expansion $(\theta)$ so that we have [37] $a_{2}=a_{3}^{m}$

(ii) $T+\bar{T}=\rho_{m}+\rho_{\lambda}-3 p_{\lambda}=0$

which physically corresponds to the vanishing of the trace of both dark matter and dark energy tensors. This is analogous to the disordered radiation condition of general relativity [38].

(iii) The special law of variation for Hubble parameter proposed by Berman [24] is taken as follows

$H=k a^{-n}$

where $k>0$ and $n \geq 0$.

These conditions are more rational in this study because of their physical characteristics and dynamical attitude. Application of these conditions along with different choices of $n$, enables us to propose the following models of the universe.

Case (I): Exponential Expansion Model: $n=0$

Substituting $n=0$ in equation (31), we get

$$
a=l_{1} e^{k t}
$$

where $l_{1}>0$ is a constant of integration.

Therefore, we have the following volume scale factor

$V=a^{3}=c_{1} e^{3 k t}$

where $c_{1}=l_{1}^{3}>0$. 
Now from equations (17), (24), (29) and (33), we obtain the expression for the metric potentials as

$$
\left.\begin{array}{l}
a_{1}=a_{2}=c_{1}^{\frac{m}{2 m+1}} e^{\frac{3 m k t}{2 m+1}} \\
a_{3}=c_{1}^{\frac{1}{2 m+1}} e^{\frac{3 k t}{2 m+1}}
\end{array}\right\}
$$

Also from equations (28), (30) and (34), we obtain the expression for BD scalar field in the model as follows

$$
\varphi=\frac{\varphi_{0}}{c_{1}} \int e^{-3 k t} d t=-\frac{\varphi_{0}}{3 c_{1} k e^{3 k t}}+\psi_{0}
$$

where $\varphi_{0}$ and $\psi_{0}$ are the constants of integration.

Now a proper choice of coordinates and constants (choosing $c_{1}=1, k=1 / 3$ and $\left.\psi_{0}=0\right)$, the metric (1) can be written, with the help of equation (34), as

$d s^{2}=d t^{2}-e^{\frac{2 m t}{2 m+1}}\left(d x^{2}+e^{-2 x} d y^{2}\right)-e^{\frac{2 t}{2 m+1}} d z^{2}$

with the BD scalar field in this model given by

$\varphi=\frac{\varphi_{0}}{e^{t}}$

Now we present some physical and kinematical properties for this model. Equations (36) and (37) represent Bianchi type-III holographic dark energy model in BD scalar-tensor theory of gravitation. We figure out some physical and kinematical parameters that play a vital role in the discussion of cosmology.

Spatial volume is found as

$V=e^{t}$

The average Hubble's parameter is given by

$H=k=\frac{1}{3}$

The scalar expansion is found as

$\theta=3 H=1$ 
The shear scalar is

$$
\sigma^{2}=\frac{(m-1)^{2}}{2(2 m+1)^{2}}
$$

The average anisotropy parameter is

$$
\Delta=\frac{3(m-1)^{2}}{(2 m+1)^{2}}
$$

The deceleration parameter is obtained as $q=-1$

Now using equations (36) and (37) in equation (25) we get the holographic pressure as

$$
8 \pi \varphi^{-1} p_{\lambda}=\frac{2(m+1)(2 m+1)-\left(m^{2}+m+1\right)-(2 m+1)^{2}(\omega+2)}{2(2 m+1)^{2}}
$$

where $\varphi$ is given by equation (37).

Using equation (36) in equation (15), we obtain the energy density of dark matter as

$$
\rho_{m}=\rho_{0} e^{-t}=\frac{\rho_{o}}{e^{t}}
$$

where $\rho_{0}>0$ is a real constant of integration. Now using equations (45), (36), (37) in equation (27), we get the energy density of holographic dark energy as

$$
8 \pi \varphi^{-1} \rho_{\lambda}=\frac{2 m(m+2)-(2 m+1)^{2}(\omega+2)+16 \pi(2 m+1)^{2} \varphi_{\circ}^{-1} \rho_{\circ}-2(2 m+1)^{2} e^{-\frac{2 m t}{2 m+1}}}{2(2 m+1)^{2}}
$$

where $\varphi$ is given by equation (37).

With the use of equations (44) and (46) in equation (13), we have the equation of state $(\mathrm{EoS})$ parameters as

$$
w=\frac{p_{\lambda}}{\rho_{\lambda}}=\frac{2(m+1)(2 m+1)-\left(m^{2}+m+1\right)-(2 m+1)^{2}(\omega+2)}{2 m(m+2)-(2 m+1)^{2}(\omega+2)+16 \pi(2 m+1)^{2} \varphi_{\circ}^{-1} \rho_{\circ}-2(2 m+1)^{2} e^{-\frac{2 m t}{2 m+1}}}
$$


which shows that $w$ is a function of cosmic time $t$. By using equation (47), the variation of the equation of state $(\mathrm{EoS})$ parameter $(w)$ for dark energy with time $(t)$ is depicted in Figure 1 for $n=0$ corresponding to $q=-1$ (exponential expansion). It is observed that the value of $w$ lies in the quintessence region $(w>-1)$ and $w \rightarrow 0$ when $t \rightarrow \infty$ and this negative value of $w$ is supported by SN Ia data [5]. On the contrary, in the context of general relativity, Yadav et al. [39] studied dark energy model with variable equation of state parameter $(w)$ using law of variation of Hubble's parameter and they found the range of $w$ as $-1.6<w<-1$ (phantom fluid dominant universe). From these data, it reveals that the universe at early stage was dominated by quintessence fluid and it will be dustfilled universe at late time which is also realistic.

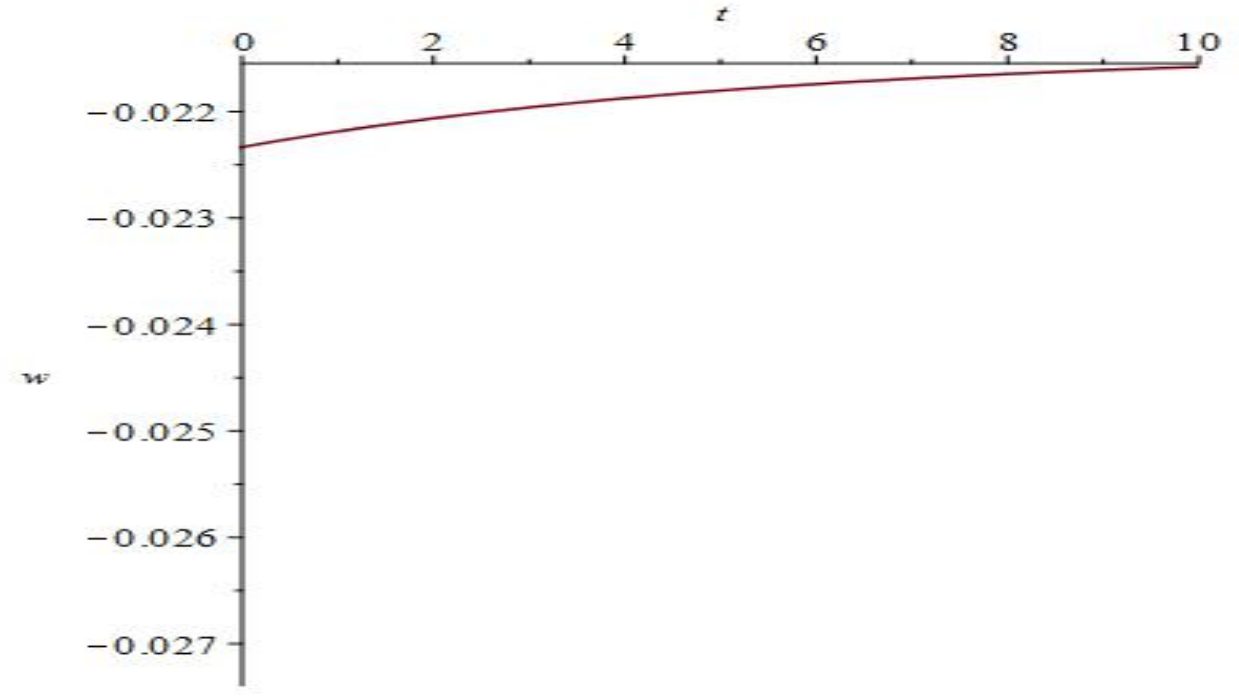

Figure 1. The plot of EoS parameter $(w)$ vs. cosmic time $(t)$ for $n=0$

$$
\left(m=0.1, \omega=0.1, \rho_{0}=\varphi_{0}=1\right)
$$


50 Mohammad Moksud Alam

The coincidence parameter is

$$
r=\frac{\rho_{\lambda}}{\rho_{m}}=\frac{(2 m+1)^{2}(\omega+2)-2 m(m+2)+2(2 m+1)^{2} e^{-\frac{2 m t}{2 m+1}}}{16 \pi \rho_{0}^{-1} \rho_{0}(2 m+1)^{2}}-1
$$

The matter density parameter $\Omega_{m}$ and the holographic dark energy density parameter $\Omega_{\lambda}$ are given by

$$
\Omega_{m}=\frac{\rho_{m}}{3 H^{2}}, \quad \Omega_{\lambda}=\frac{\rho_{\lambda}}{3 H^{2}}
$$

Using equations (39), (45) and (46) in (49) we obtain the overall density parameters as

$$
\Omega=\Omega_{m}+\Omega_{\lambda}=\frac{3(2 m+1)^{2}(\omega+2)-6 m(m+2)+6(2 m+1)^{2} e^{-\frac{2 m t}{2 m+1}}}{16 \pi \varphi_{0}^{-1}(2 m+1)^{2} e^{t}}
$$

Moreover, the jerk parameter is calculated as

$$
j(t)=\frac{1}{H^{3}} \frac{\dddot{a}}{a}=1
$$

We notice that this value overlaps with flat $\Lambda C D M$ models.

It may be observed in this case that there is exponential spatial volume expansion with time. The mean Hubble parameter, scalar expansion and shear scalar are found to be constants from equations (39), (40) and (41), respectively. Also $\rho_{m}, \rho_{\lambda}$ and $p_{\lambda}$ are constants at $t=0$ while they all vanish for infinitely large time. The coincidence parameter $r$ remains constant at $t=0$ and $t \rightarrow \infty$ as in [28] for the similar case. The average density is constant when $t=0$ and vanishes when $t \rightarrow \infty$ . The universe exhibits exponential expansion since $q=-1$.

\section{Case (II): Power-law Expansion Model: $n \neq 0$}

For $n \neq 0$, Equation (31) gives

$a=\left(n k t+c_{2}\right)^{\frac{1}{n}}$

where $c_{2}$ is an arbitrary constant of integration. 
Therefore, the volume scale factor is given by

$$
V=a^{3}=\left(n k t+c_{2}\right)^{\frac{3}{n}}
$$

Now, from equations (17), (24), (29) and (53), we obtain the expression for the metric potentials as

$$
\left.\begin{array}{l}
a_{1}=a_{2}=\left(n k t+c_{2}\right)^{\frac{3 m}{n(2 m+1)}} \\
a_{3}=\left(n k t+c_{2}\right) \frac{3}{n(2 m+1)}
\end{array}\right\}
$$

Also, from equations (28), (30) and (34), we obtain the expression for the BD scalar field in the model as follows

$$
\varphi=\frac{\varphi_{0}}{k(n-3)}\left(n k t+c_{2}\right)^{\frac{n-3}{n}}+\psi_{0}
$$

where $\varphi_{0}$ and $\psi_{0}$ are constants of integration.

Now through a proper choice of coordinates and constants (choosing $c_{2}=0, n k=1$ and $\psi_{0}=0$ ), the metric (1) can be written, with the help of equation (54), as

$$
d s^{2}=d t^{2}-t^{\frac{6 m}{n(2 m+1)}}\left(d x^{2}+e^{-2 x} d y^{2}\right)-t^{\frac{6}{n(2 m+1)}} d z^{2}
$$

and the BD scalar field is given by

$$
\varphi=\frac{\varphi_{0} n}{n-3} t^{\frac{n-3}{n}}
$$

where strictly $n \neq 3$.

As before, here, we discuss some physical and kinematical properties of this model. Equation (56) along with equation (57) represents Bianchi type-III holographic dark energy model in BD scalar-tensor theory of gravitation. The following are the physical and kinematical parameters which play a vital role in the discussion of cosmology.

Spatial volume is found as

$V=t^{\frac{3}{n}}$ 
The average Hubble's parameter is given by

$H=\frac{1}{n t}$

The scalar expansion is found as

$\theta=3 H=\frac{3}{n t}$

The shear scalar is

$\sigma^{2}=\frac{9(m-1)^{2}}{2 n^{2}(2 m+1)^{2} t^{2}}$

The average anisotropy parameter is

$\Delta=\frac{3(m-1)^{2}}{(2 m+1)^{2}}$

The deceleration parameter is obtained as

$q=n-1$

Now, using equations (56) and (57) in equation (25), we get the holographic pressure as

$8 \pi \varphi^{-1} p_{\lambda}=\frac{18 m(m+2)+(2 m+1)^{2}(n-3)(6-n \omega+3 \omega)}{2 n^{2}(2 m+1)^{2} t^{2}}$

where $\varphi$ is given by equation (57).

Using equation (56) in equation (15), we obtain the energy density of dark matter as

$\rho_{m}=\rho_{0} t^{-\frac{3}{n}}$

where $\rho_{0}>0$ is a real constant of integration. Now, using equations (65), (56) and (57) in equation (27), we get the energy density of holographic dark energy as

$8 \pi \rho^{-1} \rho_{\lambda}=\frac{18 m(m+2)+(2 m+1)^{2}(n-3)(6-n \omega+3 \omega)}{2 n^{2}(2 m+1)^{2} t^{2}}-t^{-\frac{6 m}{(2 m+1) n}}-8 \pi \rho_{0}^{-1} \rho_{0} \frac{n-3}{n t}$

where $\varphi$ is given by equation (57). 
Using equations (64) and (66) in equation (13), we have the equation of state (EoS) parameter as

$$
\begin{aligned}
w=\frac{p_{\lambda}}{\rho_{\lambda}} & =\left\{18 m(m+2)+(2 m+1)^{2}(n-3)(6-n \omega+3 \omega)\right\} \\
& \times\left\{18 m(m+2)+(2 m+1)^{2}(n-3)(6-n \omega+3 \omega)\right. \\
& \left.-2(2 m+1)^{2} n^{2} t^{\frac{4 m n-6 m+2 n}{(2 m+1) n}}-16 \pi \varphi_{0}^{-1} \rho_{0}(2 m+1)^{2}(n-3) n t\right\}
\end{aligned}
$$

which shows that $w$ is a function of cosmic time $t$. By using equation (67), the variation of the equation of state $(\mathrm{EoS})$ parameter $(w)$ for dark energy with time $(t)$ is shown in Figure 2 for $n=0.5$ corresponding to $q=-0.5$ (accelerating expansion i.e. $-1<q<0$ ). It is seen that the $\operatorname{EoS}$ parameter $w$, at the very early stage of the universe, is found to be positive but surprisingly, changing the course of evolution, it goes to the phantom region $(w<-1)$ and then enters into the quintessence region $(w>-1)$ evolving with negative value. Yadav et al. [39] obtained the range of $w$ as $-1.1<w<-0.5$ throughout the evolution and our result shows $-1.1<w<0$ for the similar case with dissimilarity at the very early epoch of the evolution. This dissimilarity leads to an interesting point which may accommodate stiff fluid filled universe [40]. 
54 Mohammad Moksud Alam

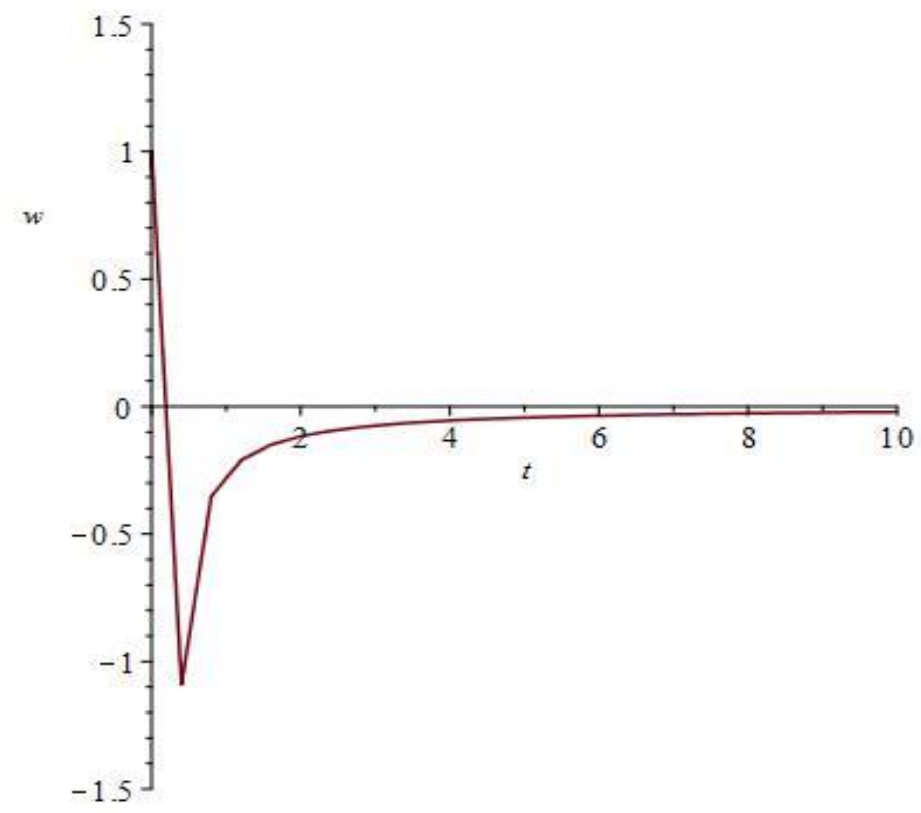

Figure 2. The plot of EoS parameter $(w)$ vs. cosmic time $(t)$ for $n=0.5$

$$
\left(m=0.1, \omega=0.1, \rho_{0}=\varphi_{0}=1\right)
$$

The coincidence parameter is given by

$$
r=\frac{\rho_{\lambda}}{\rho_{m}}=\frac{18 m(m+2)+(2 m+1)^{2}(n-3)(6-n \omega+3 \omega)}{16 \pi \rho_{0}{ }^{-1} \rho_{0}(2 m+1)^{2}(n-3) n t}-\frac{n t^{\frac{2 m n-6 m+n}{(2 m+1) n}}}{8 \pi \rho_{0}{ }^{-1} \rho_{0}(n-3)}-1
$$

Using equations (59), (65), (66) in (49), we obtain the overall density parameters as

$$
\begin{aligned}
\Omega=\frac{\rho}{3} n^{2} t^{\frac{2 n-3}{n}} & +\frac{\varphi_{0} n}{8 \pi(n-3)}\left[\frac{18 m(m+2)+(2 m+1)^{2}(n-3)(6-n \omega+3 \omega)}{6(2 m+1)^{2}}\right. \\
& \left.-\frac{n^{2}}{3} t^{\frac{4 m n-6 m+2 n}{(2 m+1) n}}-\frac{8 \pi \rho_{0}}{3 \varphi_{0}}(n-3) n t\right] t^{\frac{n-3}{n}}
\end{aligned}
$$


Now we compute the jerk parameter for this model as

$$
j(t)=\frac{1}{H^{3}} \frac{\dddot{a}}{a}=(n-1)(2 n-1)
$$

Which also overlaps with flat $\Lambda C D M$ models for $n=3 / 2$. The jerk parameter vanishes for $n=1$ or $n=1 / 2$.

The above results enable us to study the behavior of the physical and kinematical parameters of the universe. The model, given by equation (56), has an initial singularity. The spatial volume $V$ of the model increases with time showing the spatial expansion of the universe. It can be seen that the parameters $H, \theta, \sigma^{2}, \rho_{\lambda}, p_{\lambda}$ and $\varphi$ are infinite at $t=0$ and these all vanish when $t \rightarrow \infty$. The coincidence parameter $r$ tends to infinity when $t=0$ and $r \rightarrow-1$ as $t \rightarrow \infty$. The overall density parameter $\Omega$ decreases with the evolution of time $t$. The present model retains the anisotropy except when $m=1$, otherwise the universe is isotropic and shear free. The deceleration parameter takes the range $-1<q<0$ for $0<n<1$ and thus the universe accelerates, otherwise it decelerates in the standard way.

\section{Conclusions}

We investigated spatially homogeneous and anisotropic Bianchi type-III minimally interacting holographic dark energy models in the scalar-tensor theory of gravitation. Using this theory, we presented two models, namely exponential expansion model and power law expansion model. In these models, we derived the energy density of matter, holographic dark energy density, holographic pressure, equation of state parameter, the scalar field in the models, total energy density in the universe, the coincidence and jerk parameter and discussed their physical behavior accordingly. We also showed, the universe will be shear free and isotropic when $m=1$, for both of the models and the dark energy equation of state (EoS) parameter, for these models, is found to be negative with an exception in power law expansion model at the very early epoch of the evolution that reveals an interesting phenomenon. The EoS parameter in the proposed models may play a role of a 
56 Mohammad Moksud Alam

candidate of the dark energy. In fine, it is obvious that the transition of the dark energy equation of state can be contained by the minimally interacting holographic dark energy model.

\section{Acknowledgement}

The author would like to thank the learned reviewer for constructive comments which have improved the presentation of this work.

\section{References}

[1] S. Perlmutteret al.: Astrophys. J.,1999, 517, 565.

[2] A. G. Riesset al.: Astron. J., 1998, 116, 1009.

[3] C. L. Bennetet al.: Astrophys. J. Suppl. Ser., 2003, 148, 1.

[4] D. N. Speergel et al.: J. Suppl. Ser., 2003, 148, 175.

[5] M. Tegmarket al.: Astrophys. J., 2004, 606, 702.

[6] E. J. Copeland et al.: Int. J. Mod. Phys. D, 2006, 15, 1753.

[7] S. Nojiri, S. D. Odintsov: Phys. Rev. D, 2003, 68, 023512.

[8] C. H. Brans, R. H. Dicke: Phys. Rev. D, 1961, 124, 925.

[9] D. Saez, V. J. Ballester: Phys. Lett. A, 1986, 113, 467.

[10] S. M. Carroll et al.: New J. Phys., 2006, 8, 1367.

[11] T. Harko, F. S. N. Lobo, S. Nojiri, S. D. Odintsov: Phys. Rev.D, 2011, 84, 024020.

[12] O. Bertolami, P. J. Martins.: Phys. Rev. D, 2000, 61, 0604007.

[13] N. Banerjee, D. Pavon: Phys. Rev. D, 2001, 63, 043504.

[14] C. Mathiazhagan, V. B. Johri: Class. Quantum Gravity,1984, 1, 129.

[15] L. O. Pimentel:Mod. Phys. Lett. A, 1997, 12, 25, 1865.

[16] A. Linde: Phys. Lett. B, 1990, 238, 160.

[17] B. Guberina et al.: J. Cosmol. Astropart. Phys., 2007, 01, 012.

[18] A. G. Cohen et al.: Phys. Rev. Lett., 1999, 82, 4971.

[19] P. Horova, D. Minic: Phys. Rev. Lett., 2000, 85, 1610.

[20] S. Thomas: Phys. Rev. Lett., 2002, 89, 081301.

[21] S. D. H. Hsu.: Phys. Lett. B, 2004, 594, 13.

[22] M. Li: Phys. Lett. B, 2004, 603, 1.

[23] M. R. Setare, E. C. Vagenas: Int. J. Mod. Phys. D, 2009, 18, 147. 
[24] S. Das, A. A. Mammon.: Astrophys. Space Sci., 2014, 351, 651.

[25] S. Sarkar, C.R. Mahanta: Int. J. Theor. Phys., 2013, 52, 1482.

[26] S. Sarkar: Astrophys. Space Sci., 2014, 352, 859.

[27] S. Sarkar: Astrophys. Space Sci., 2014, 349, 985.

[28] K. S. Adhav et al.: Astrophys. Space Sci., 2014, 353, 249.

[29] M. Kiran, D. R. K. Reddy, V. U. M. Rao.: Astrophys Space Sci, 2014, 354, 577.

[30] M. M. Alam: Bianchi Types Anisotropic Cosmological Model in General Relativity, M. Phil Thesis, CU, 2018.

[31] S. Umadevi, G. Ramesh: Astrophys Space Sci., 2015, 359, 51.

[32] M. Kiran et al.: Astrophys Space Sci, 2015, 356, 407.

[33] B. Bertotti, L. Iess and P. Tortora: Nature, 2003, 425, 374.

[34] A. D. Felice et al.: Phys. Rev. D, 2006, 74, 103005.

[35] C. J. A. P. Martins: Phil. Trans. Roy. Soc. Lond. A, 2002, 360, 2681.

[36] M. S. Berman.: NuovoCimento B, 1983, 74, 182.

[37] C. B. Collinset al.: Gen. Relativ. Gravit, 1983, 12, 805.

[38] T. Vidyasagar et al.: Eur. Phys. J. Plus, 2014, 129, 36.

[39] A. K. Yadavet al.: Int J Theor Phys., 2011, 50, 871.

[40] P. H. Chavanis: Phys. Rev. D, 2015, 92, 103004.

The Chittagong Univ. J. Sc. Vol. 42(1), 2020 